\title{
Magnetic field of an in-plane vortex inside and outside a layered superconducting film
}

\author{
Edson Sardella \\ Departamento de Física, Faculdade de Ciências, Universidade Estadual Paulista, Caixa Postal 473, 17033-360, Bauru-SP, Brazil
}

(Received 10 February 2000)

\begin{abstract}
In the present work we study an anisotropic layered superconducting film of finite thickness. The film surfaces are considered parallel to the $b c$ face of the crystal. The vortex lines are oriented perpendicular to the film surfaces and parallel to the superconducting planes. We calculate the local field and the London free energy for this geometry. Our calculation is a generalization of previous works where the sample is taken as a semi-infinite superconductor. As an application of this theory we investigate the flux spreading at the superconducting surface.
\end{abstract}

Scanning superconducting quantum interference device (SQUID) microscopy has been used to image interlayer Josephson vortices trapped between the planes of layered superconductors. This technique has been used to measure the out-plane London penetration depth that gives the distance over which the interlayer current $j_{c}$ changes as a function of in-plane coordinates. ${ }^{1,2}$ These measurements have been important to test the interlayer tunneling model as a candidate to explain the mechanism of superconductivity for the high$T_{c}$ superconductors. ${ }^{3,4}$

Recently, Kirtley, Kogan, Clem, and Moler ${ }^{5}$ have found expressions for the local magnetic field emerging from a superconductor with the vortex lines parallel to the planes, and normal to a crystal face. Their geometry consists of a semiinfinite anisotropic superconductor. Furthermore, they have used these expressions to fit the experimental data at the surface in order to obtain an estimate of the value of the out-plane penetration depth $\lambda_{c}$. They have shown that, neglecting the vortex spreading at the surface may overestimate $\lambda_{c}$ as much as $30 \%$.

In the present paper we extend the work of Ref. 5 to an anisotropic layered superconducting film of finite thickness and of infinite extent in the $b c$ face of the crystal. We will show that, if the thickness of the film is of order or smaller than $\lambda_{c}$, the magnetic field distribution is even more affected by flux spreading.

Let us first formulate the problem to be solved. The geometry we consider is illustrated in Fig. 1. We suppose that the vortex line is perpendicular to the film. We will calculate the local field inside the film using the London equation. For this geometry this equation is given by

$$
\boldsymbol{\nabla} \times[\overleftrightarrow{\nabla} \times \mathbf{h}]+\mathbf{h}=\hat{\mathbf{z}} \Phi_{0} \delta(\mathbf{r})
$$

where $\overleftrightarrow{\nabla}$ is the London (tensor) penetration depth. This tensor is diagonal and its components are given by $\Lambda_{x x}=\Lambda_{y y}$ $=\lambda_{c}^{2}, \Lambda_{z z}=\lambda_{a b}^{2}$; here $\lambda_{a b}$ and $\lambda_{c}$ are the in- and out-plane penetration depth respectively; $\Phi_{0}$ is the quantum flux. The film is anisotropic along the $c$ direction.

Outside the sample, the local field satisfies the equation

$$
\nabla^{2} \mathbf{h}=0
$$

Although we will consider the case of a single vortex, the generalization to the case of $N$ vortices is straightforward. To proceed is more convenient to Fourier transform Eqs. (1) and (2). For $|z|<d / 2$, using the Maxwell equation $\boldsymbol{\nabla} \cdot \mathbf{h}=0$, we obtain a set of three coupled differential equations for the two dimensional Fourier transform of the local magnetic field $\mathbf{h}(\mathbf{k}, z)=\int d^{2} r e^{-\imath \mathbf{k} \cdot \mathbf{r}} \mathbf{h}(\mathbf{r}, z)$,

$$
\begin{gathered}
{\left[1+\lambda_{a b}^{2} k^{2}-\lambda_{a b}^{2} \frac{\partial^{2}}{\partial z^{2}}\right] h_{x}=0} \\
{\left[1+\lambda_{a b}^{2} k^{2}-\lambda_{c}^{2} \frac{\partial^{2}}{\partial z^{2}}\right] h_{y}+\left(\lambda_{c}^{2}-\lambda_{a b}^{2}\right) i k_{y} \frac{\partial h_{z}}{\partial z}=0} \\
{\left[1+\lambda_{a b}^{2} k_{x}^{2}+\lambda_{c}^{2} k_{y}^{2}-\lambda_{a b}^{2} \frac{\partial^{2}}{\partial z^{2}}\right] h_{z}+\left(\lambda_{c}^{2}-\lambda_{a b}^{2}\right) i k_{y} \frac{\partial h_{y}}{\partial z}=\Phi_{0}}
\end{gathered}
$$

For $|z|>d / 2$ one has

$$
\left(\frac{\partial^{2}}{\partial z^{2}}-k^{2}\right) \mathbf{h}=0
$$

At the vacuum-superconductor interfaces $z= \pm d / 2$ the field components are continuous and the component of the current perpendicular to both film surfaces vanishes. One has

$$
\begin{gathered}
\mathbf{h}_{<}(\mathbf{k},-d / 2)=\mathbf{h}_{m}(\mathbf{k},-d / 2), \\
\mathbf{h}_{m}(\mathbf{k}, d / 2)=\mathbf{h}_{>}(\mathbf{k}, d / 2), \\
\hat{\mathbf{z}} \cdot\left[\mathbf{D}_{z}(\mathbf{k}) \times \mathbf{h}_{m}\right]_{z= \pm d / 2}=0, \\
\mathbf{D}_{z}(\mathbf{k}) \cdot \mathbf{h}=0,
\end{gathered}
$$

where the operator $\mathbf{D}_{z}(\mathbf{k})=i \mathbf{k}+\hat{\mathbf{z}}(\partial / \partial z)$. The subscripts $(<,>)$ stand for below the surface $z=-d / 2$ and above the surface $z=d / 2$, respectively, whereas the subscript $m$ is meant for the field inside the sample.

We start by solving first Eq. (6). The solution which satisfies the boundary condition of Eq. (10) takes the form 


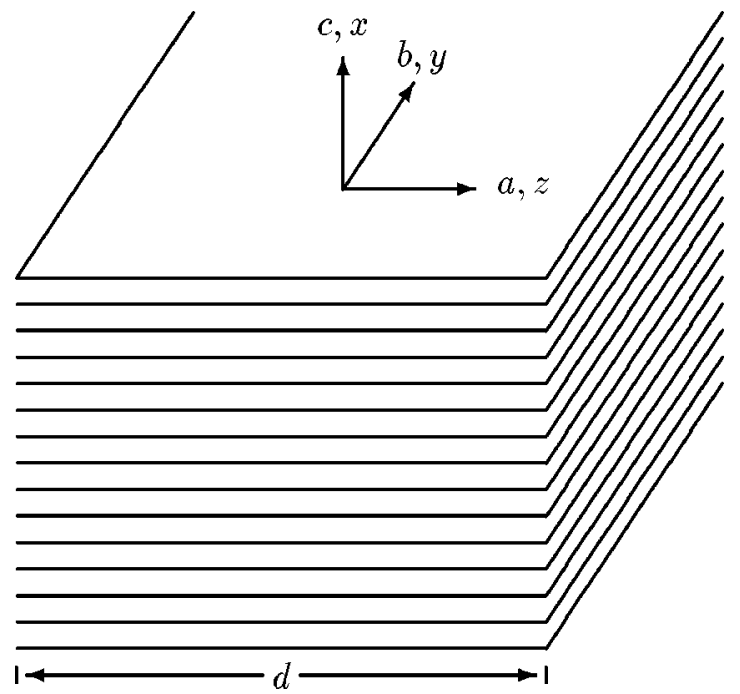

FIG. 1. Geometry of the film used in this work. The vortex lines are oriented perpendicular to the $b c$ face of the crystal.

$$
\begin{gathered}
\mathbf{h}_{>}(\mathbf{k}, z)=(-i \mathbf{k}+\hat{\mathbf{z}} k) \varphi(\mathbf{k}) e^{-k(z-d / 2)}, \\
\mathbf{h}_{<}(\mathbf{k}, z)=(i \mathbf{k}+\hat{\mathbf{z}} k) \varphi(\mathbf{k}) e^{k(z+d / 2)},
\end{gathered}
$$

where $\varphi(\mathbf{k})$ is a scalar function which will be determined by using the boundary condition either of Eq. (7) or (8).

Equation (3) can also be easily solved. We have

$$
h_{m, x}(\mathbf{k}, z)=W_{1} e^{\alpha z}+W_{2} e^{-\alpha z}
$$

where

$$
\alpha=\sqrt{\frac{1+\lambda_{a b}^{2} k^{2}}{\lambda_{a b}^{2}}},
$$

and the $W$ 's are two constants to be determined by using the boundary conditions.

The other two components of the local field can be determined by decoupling Eqs. (4) and (5). This can be done by calculating the determinant of the matrix formed by the coefficients of Eqs. (4) and Eq. (5). This yields the following equation for $h_{m, y}$ :

$$
\left[1+\lambda_{a b}^{2} k^{2}-\lambda_{a b}^{2} \frac{\partial^{2}}{\partial z^{2}}\right]\left[1+\lambda_{a b}^{2} k_{x}^{2}+\lambda_{c}^{2} k_{y}^{2}-\lambda_{c}^{2} \frac{\partial^{2}}{\partial z^{2}}\right] h_{m, y}=0
$$

The solution for this equation is given by

$$
h_{y, m}(\mathbf{k}, z)=W_{3} e^{\alpha z}+W_{4} e^{-\alpha z}+W_{5} e^{\gamma z}+W_{6} e^{-\gamma z},
$$

where the $W$ 's are constants to be determined by using the boundary conditions and

$$
\gamma=\sqrt{\frac{1+\lambda_{a b}^{2} k_{x}^{2}+\lambda_{c}^{2} k_{y}^{2}}{\lambda_{c}^{2}}}
$$

The solution for $h_{m, z}$ can be found by inserting Eq. (16) back into Eq. (4) or (5). One has

$$
\begin{aligned}
h_{m, z}(\mathbf{k}, z)= & \frac{\Phi_{0}}{\lambda_{c}^{2} \gamma^{2}}+\frac{\alpha}{i k_{y}}\left(W_{3} e^{\alpha z}-W_{4} e^{-\alpha z}\right) \\
& -\frac{i k_{y}}{\gamma}\left(W_{5} e^{\gamma z}-W_{6} e^{-\gamma z}\right) .
\end{aligned}
$$

The determination of the constants $W_{i}$ is very cumbersome and we omit it here. We just present the main steps of the complete solution. First of all, we use the Maxwell equation (10). This allows us to write $W_{3}$ and $W_{4}$ in terms of $W_{1}$ and $W_{2}$. Secondly, we use the boundary condition of Eq. (9) in both faces of the film. This leads us to the solution of $W_{5}$ and $W_{6}$ in terms of $W_{1}$ and $W_{2}$. Then, we are left only with three constants to determine, namely, $W_{1}, W_{2}$, and $\varphi$. Thirdly, we use the continuity of the local field at the film surfaces [either Eq. (7) or (8); both of them yields the same solution to these constants]. One obtains

$$
\begin{gathered}
W_{1}=-i k_{x} \frac{\varphi}{2 \sinh \left(\frac{\alpha d}{2}\right)}, \\
W_{2}=i k_{x} \frac{\varphi}{2 \sinh \left(\frac{\alpha d}{2}\right)},
\end{gathered}
$$

$$
W_{3}=\frac{\lambda_{a b}^{2} k_{x} k_{y}}{1+\lambda_{a b}^{2} k_{x}^{2}} W_{1}
$$

$$
W_{4}=\frac{\lambda_{a b}^{2} k_{x} k_{y}}{1+\lambda_{a b}^{2} k_{x}^{2}} W_{2}
$$

$$
\begin{aligned}
W_{5}= & \frac{k_{y}}{k_{x}\left(1+\lambda_{a b}^{2} k_{x}^{2}\right) \sinh (\gamma d)} \\
& \times\left\{W_{1} \sinh \left[(\gamma+\alpha) \frac{d}{2}\right]+W_{2} \sinh \left[(\gamma-\alpha) \frac{d}{2}\right]\right\},
\end{aligned}
$$

$$
\begin{aligned}
W_{6}= & \frac{k_{y}}{k_{x}\left(1+\lambda_{a b}^{2} k_{x}^{2}\right) \sinh (\gamma d)}\left\{W_{1} \sinh \left[(\gamma-\alpha) \frac{d}{2}\right]\right. \\
& \left.+W_{2} \sinh \left[(\alpha+\gamma) \frac{d}{2}\right]\right\},
\end{aligned}
$$

$$
\varphi(\mathbf{k})=\frac{\Phi_{0}}{\lambda_{c}^{2} \gamma^{2}} \Delta(\mathbf{k})
$$

where

$$
\Delta(\mathbf{k})=\left[k+\frac{\lambda_{a b}^{2} k_{x}^{2} \alpha \operatorname{coth}\left(\frac{\alpha d}{2}\right)+\frac{k_{y}^{2}}{\gamma} \operatorname{coth}\left(\frac{\gamma d}{2}\right)}{1+\lambda_{a b}^{2} k_{x}^{2}}\right]^{-1} .
$$



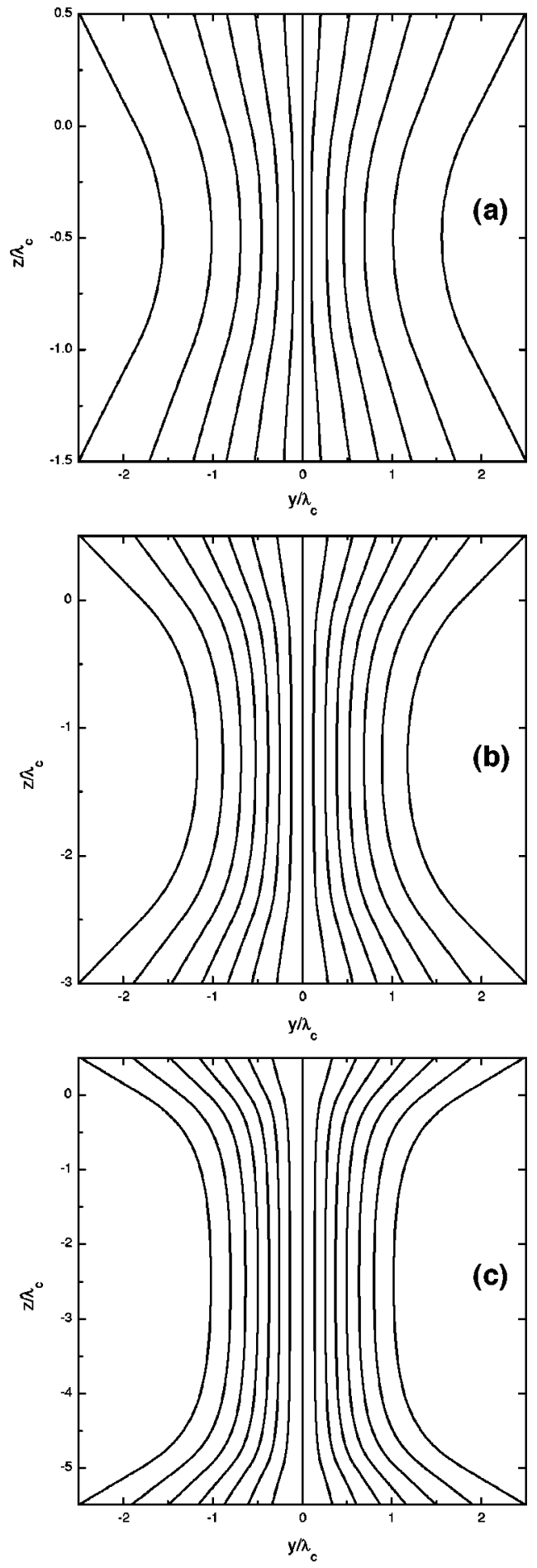

FIG. 2. Streamline mapping of the integrated field $\overrightarrow{\mathcal{H}}$, for an anisotropic superconducting film. The spacing of the streamlines is proportional to $\left(\partial \mathcal{H}_{z} / \partial y\right)^{-1}$ at $z=-\left(d+0.5 \lambda_{c}\right)$. (a) shows the streamlines for a film of thickness $d=\lambda_{c}$, (b) for $d=2.5 \lambda_{c}$, and (c) for $d=5 \lambda_{c}$.

Finally, upon substituting Eqs. (19)-(25) into Eqs. (13), (16) and (18), we find for the local magnetic field inside the film

$$
h_{m, x}(\mathbf{k}, z)=-i k_{x} \varphi(\mathbf{k}) \frac{\sinh (\alpha z)}{\sinh \left(\frac{\alpha d}{2}\right)},
$$

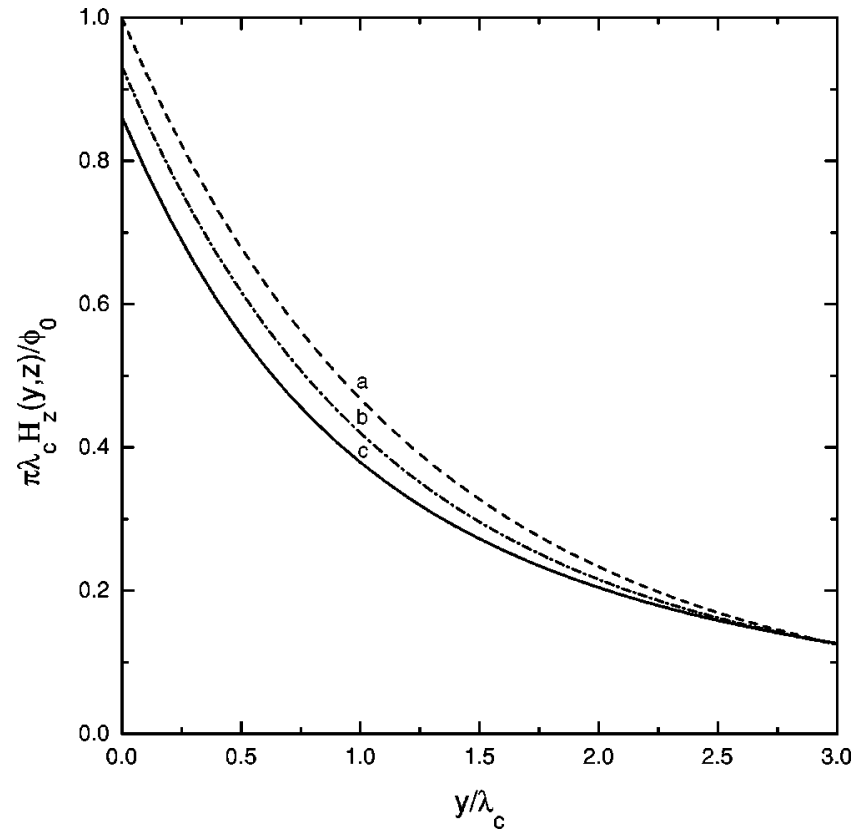

FIG. 3. The $z$ component of the integrated field as a function of $y$ at $z=0$ for three different values of the film thickness: (a) $d$ $=5 \lambda_{c}$ (dashed line); (b) $d=1.5 \lambda_{c}$ (dot-dashed line); (c) $d=\lambda_{c}$ (continuous line).

$$
\begin{gathered}
h_{m, y}(\mathbf{k}, z)=-i k_{y} \frac{\varphi(\mathbf{k})}{1+\lambda_{a b}^{2} k_{x}^{2}}\left[\lambda_{a b}^{2} k_{x}^{2} \frac{\sinh (\alpha z)}{\sinh \left(\frac{\alpha d}{2}\right)}+\frac{\sinh (\gamma z)}{\sinh \left(\frac{\gamma d}{2}\right)}\right], \\
h_{m, z}(\mathbf{k}, z)=\frac{\Phi_{0}}{\lambda_{c}^{2} \gamma^{2}}-\frac{\varphi(\mathbf{k})}{1+\lambda_{a b}^{2} k_{x}^{2}}\left[\lambda_{a b}^{2} k_{x}^{2} \alpha \frac{\cosh (\alpha z)}{\sinh \left(\frac{\alpha d}{2}\right)}\right. \\
\left.+\frac{k_{y}^{2}}{\gamma} \frac{\cosh (\gamma z)}{\sinh \left(\frac{\gamma d}{2}\right)}\right] .
\end{gathered}
$$

We would like to point out that these results could not be obtained from those of Ref. 5 without solving the problem. In fact, the solution of the London equation for a superconducting film is different and more difficult than for a semiinfinite superconductor.

Let us turn our discussion to the calculation of the London free energy. The energy of the vortex system is given by $F$ $=F_{V}+F_{S}$, where $F_{V}$ is the field energy in the vacuum and $F_{S}$ is the energy inside the superconductor. One has

$$
\begin{gathered}
F_{V}=\frac{1}{8 \pi} \int \frac{d^{2} k}{(2 \pi)^{2}}\left\{\int_{d / 2}^{\infty} d z\left|h_{>}(\mathbf{k}, z)\right|^{2}\right. \\
\left.\quad+\int_{-\infty}^{-d / 2} d z\left|h_{<}(\mathbf{k}, z)\right|^{2}\right\}, \\
F_{S}=\frac{1}{8 \pi} \int \frac{d^{2} k}{(2 \pi)^{2}} \int_{-d / 2}^{d / 2} d z\left\{\left|h_{m}(\mathbf{k}, z)\right|^{2}+\left[\mathbf{D}_{z}(\mathbf{k})\right.\right. \\
\left.\left.\quad \times \mathbf{h}_{m}(\mathbf{k}, z)\right] \cdot \vec{\nabla} \cdot\left[\mathbf{D}_{z}(-\mathbf{k}) \times \mathbf{h}_{m}(-\mathbf{k}, z)\right]\right\} .
\end{gathered}
$$


By substituting the appropriate expressions of the local magnetic field inside Eqs. (30) and (31), after a length algebra, we obtain

$$
F=\frac{\Phi_{0}^{2}}{8 \pi} \int \frac{d^{2} k}{(2 \pi)^{2}} \frac{1}{\lambda_{c}^{2} \gamma^{2}}\left[d+2 \frac{\Delta(\mathbf{k})}{\lambda_{c}^{2} \gamma^{2}}\right] .
$$

The free energy can be generalized to an ensemble of $N$ interacting vortex lines upon multiplying the integrand of Eq. (32) by $|S(\mathbf{k})|^{2}$ where the structure factor is given by

$$
S(\mathbf{k})=\sum_{i} e^{i \mathbf{k} \cdot \mathbf{R}_{i}}
$$

Here $\mathbf{R}_{i}$ is the position of the $i$-vortex line. Note that this extended result should be valid for an ensemble of distorted vortices, that is, the positions of the vortices do not necessarily correspond to the equilibrium configuration. The first term inside Eq. (32) represents the interaction energy of the vortex lines as if the surfaces were absent. The second term represents the surface energy associated to the magnetic energy of the stray field at the superconductor-vacuum interface. Notice that for $k$ small (large $r$ ), $\gamma^{2} \sim 1 / \lambda_{c}^{2}$, and $\Delta(\mathbf{k})$ $\sim 1 / k$. Thus, the surface energy goes as $\Phi_{0}^{2} / 8 \pi^{2} r$. Consequently, the interaction on the surface depends neither on the film thickness nor on the anisotropy. This is the Pearl result for vortices emerging from a semi-infinite isotropic supercondutor. ${ }^{6}$ Another interesting particular case of Eq. (32) is the limit of a very thin film $d \rightarrow 0$, and $k$ small. In this limit, from Eq. (26) it is straightforward to show that $\Delta(\mathbf{k})$ $=1 /\left[k+2\left(\lambda_{a b}^{2} k_{x}^{2}+\lambda_{c}^{2} k_{y}^{2}\right) / d\right]$. Therefore, from Eq. (32) we obtain

$$
F=E_{0} \int \frac{d k^{2}}{(2 \pi)^{2}} \frac{2 \pi d}{k \Lambda^{-1}+\left(k_{x}^{2}+\Gamma k_{y}^{2}\right)},
$$

where $E_{0}=\left(\Phi_{0} / 4 \pi \lambda_{a b}\right)^{2}, \Lambda=2 \lambda_{a b}^{2} / d$, and $\Gamma=\lambda_{c}^{2} / \lambda_{a b}^{2}$ is the anisotropy parameter. This is precisely the energy of a single vortex in very thin film first obtained by Pearl. 7,8

Now we will turn our attention to the streamlines of the integrated field over $x$. The distribution of magnetic field emerging on the surface can be probed with a SQUID pickup loop. If the SQUID probe is oriented in the $x y$ plane, the total magnetic flux will be nearly equal to the pickup loop size times 5

$$
\mathcal{H}_{z}(y, z)=\int_{-\infty}^{\infty} h_{z}(x, y, z) d x=\int_{-\infty}^{\infty} \frac{d k_{y}}{2 \pi} h_{z}\left(0, k_{y}, z\right) e^{i k_{y} y}
$$

whereas, if the SQUID probe is oriented along the $x z$ plane, the total magnetic flux is measured through the pickup loop size times

$$
\mathcal{H}_{y}(y, z)=\int_{-\infty}^{\infty} h_{y}(x, y, z) d x=\int_{-\infty}^{\infty} \frac{d k_{y}}{2 \pi} h_{y}\left(0, k_{y}, z\right) e^{i k_{y} y} .
$$

In order to compare our results with the results of Ref. 5, we will replace the vacuum-superconductor surfaces at $z$ $=0$ and $z=-d$. This can be done through the translation $z$ $\rightarrow z+d / 2$. From Eqs. (11), (12), and (27)-(29) we obtain

$$
\begin{gathered}
\mathbf{h}_{>}(\mathbf{k}, z)=(-i \mathbf{k}+\hat{\mathbf{z}} k) \varphi(\mathbf{k}) e^{-k z}, \\
\mathbf{h}_{<}(\mathbf{k}, z)=(i \mathbf{k}+\hat{\mathbf{z}} k) \varphi(\mathbf{k}) e^{k(z+d)}, \\
h_{m, x}(\mathbf{k}, z)=-i k_{x} \varphi(\mathbf{k}) \frac{\sinh \left[\alpha\left(z+\frac{d}{2}\right)\right]}{\sinh \left(\frac{\alpha d}{2}\right)},
\end{gathered}
$$

The substitution of the appropriate expressions into Eqs. (35) and (36) yields for the $z$ component of the $\overrightarrow{\mathcal{H}}$ field,

$$
\begin{gathered}
\mathcal{H}_{>}^{z}(y, z)=\frac{\Phi_{0}}{\pi \lambda_{c}} \int_{0}^{\infty} d u \frac{\cos \left(y^{\prime} \sinh u\right) e^{-z^{\prime} \sinh u}}{\cosh u+\sinh u \operatorname{coth}\left(\frac{d}{2 \lambda_{c}} \cosh u\right)}, \\
\mathcal{H}_{m, z}(y, z)=\frac{\Phi_{0}}{\pi \lambda_{c}}\left\{\frac{\pi}{2} e^{-\left|y^{\prime}\right|}-\int_{0}^{\infty} d u \tanh u \frac{\cos \left(y^{\prime} \sinh u\right)}{\cosh u+\sinh u \operatorname{coth}\left(\frac{d}{2 \lambda_{c}} \cosh u\right)} \sinh \left(\frac{d}{2 \lambda_{c}} \cosh u\right)\right.
\end{gathered},
$$




$$
\mathcal{H}_{<}^{z}(y, z)=\frac{\Phi_{0}}{\pi \lambda_{c}} \int_{0}^{\infty} d u \frac{\cos \left(y^{\prime} \sinh u\right) e^{\left(z^{\prime}+d / \lambda_{c}\right) \sinh u}}{\cosh u+\sinh u \operatorname{coth}\left(\frac{d}{2 \lambda_{c}} \cosh u\right)}
$$

where $y^{\prime}=y / \lambda_{c}$ and $z^{\prime}=z / \lambda_{c}$.

The $y$ component takes the form

$$
\begin{aligned}
& \mathcal{H}_{>}^{y}(y, z)=\frac{\Phi_{0}}{\pi \lambda_{c}} \int_{0}^{\infty} d u \frac{\sin \left(y^{\prime} \sinh u\right) e^{-z^{\prime} \sinh u}}{\cosh u+\sinh u \operatorname{coth}\left(\frac{d}{2 \lambda_{c}} \cosh u\right)},
\end{aligned}
$$

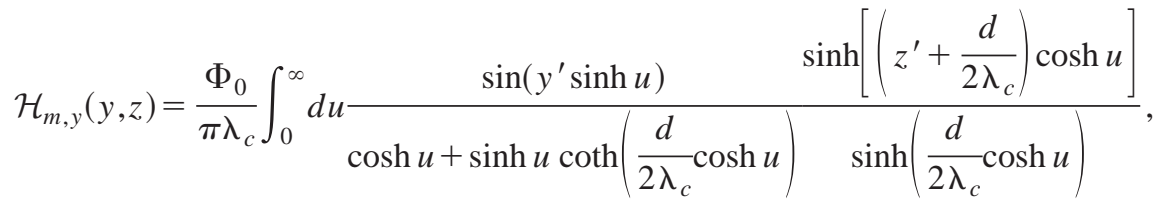

$$
\begin{aligned}
& \mathcal{H}_{<}^{y}(y, z)=-\frac{\Phi_{0}}{\pi \lambda_{c}} \int_{0}^{\infty} d u \frac{\sin \left(y^{\prime} \sinh u\right) e^{\left(z^{\prime}+d / \lambda_{c}\right) \sinh u}}{\cosh u+\sinh u \operatorname{coth}\left(\frac{d}{2 \lambda_{c}} \cosh u\right)} .
\end{aligned}
$$

Note that in the limit of $d \rightarrow \infty$, our results are exactly the same as those of Ref. 5 .

The results for the $\overrightarrow{\mathcal{H}}$ field presented above should be useful to interpret the experimental data obtained by using scanning SQUID microscopy. Unfortunately, the experiments have been performed in samples of large thickness. ${ }^{1,2}$ This renders the test of the theory impracticable. In fact, vortices have been magnetically imaged in films, but for a different geometry, that is, the superconducing planes are taken parallel to the surfaces of the film and the vortex lines are considered perpendicular to the film surfaces. ${ }^{9}$ In this case, we can extract the in-plane penetration depth $\lambda_{a b}$ rather than $\lambda_{c}$, from the fitting of the experimental data. So, we will restrict our analysis only to the theoretical expressions.

Figure 2 shows the streamlines of the $\overrightarrow{\mathcal{H}}(y, z)$ field for a single interlayer vortex centered at $x=0, y=0$. The streamlines were generated as sketched in Ref. 5 . We used various values of the film thickness. Note that as the thickness of the film grows, the flux spreading is important only near the surface, whereas deep inside the thinner film the streamlines are still very distorted, except those close to the center of the vortex.

To see how important the flux spreading inside a superconducting film is, we calculated numerically $\pi \lambda_{c} \mathcal{H}_{z}(y, z) / \Phi_{0}$ as function of $y / \lambda_{c}$ for three different val- ues of $d$ at $z=0$. As can be seen from Fig. 3, the full width at half maximum of the flux contour is $1.87 \lambda_{c}$ for the case $d=5 \lambda_{c}$, while it is $1.65 \lambda_{c}$ for $d=\lambda_{c}$. Thus, if the flux spreading inside the film is not taken into account, the value of $\lambda_{c}$ could be underestimated by $10 \%$. This error grows as the film thickness decreases.

Finally, we would like to point out that the present results agree with their isotropic counterpart. If we set $\lambda_{a b}=\lambda_{c}=\lambda$ in Eq. (25) and (26), we obtain the same result as in Ref. 9. Apparently, our results are different of those found in Ref. 10, but they show very similar streamlines.

In summary, we have calculated the field distribution of a single vortex inside and outside a layered superconducting film of arbitrary thickness. We also calculated the London free energy of an ensemble of vortices. From the expression for the energy one can recover the interaction potential between vortices for a very thin film ${ }^{7,8}$ and the vortices emerging from a semi-infinite superconductor. ${ }^{5,6}$ In addition, we have shown that flux spreading inside a superconducting film of order or smaller than $\lambda_{c}$ affects substantially the full width at half maximum of the flux contour.

The author thanks the Brazilian Agencies FAPESP and $\mathrm{CNPq}$ for financial support. The author would like to thank Professor V. G. Kogan for very useful discussions.
${ }^{1}$ K.A. Moler, J.K. Kirtley, D.G. Hinks, T.W. Li, and M. Xu, Science 279, 1193 (1998).

${ }^{2}$ J.R. Kirtley, K.A. Moler, G. Villard, and A. Maigman, Phys. Rev. Lett. 81, 2140 (1998).

${ }^{3}$ P.A. Anderson, Science 279, 1196 (1998).

${ }^{4}$ S. Chakravarty, Eur. Phys. J. B 5, 337 (1998).

${ }^{5}$ J.R. Kirtley, V.G. Kogan, J.R. Clem, and K.A. Moler, Phys. Rev. B 59, 4343 (1999).

${ }^{6}$ J. Pearl, J. Appl. Phys. 37, 4139 (1966).
${ }^{7}$ J. Pearl, Appl. Phys. Lett. 5, 65 (1964).

${ }^{8}$ V.G. Kogan, A.Yu. Simonov, and M. Ledvij, Phys. Rev. B 48, 392 (1993). In this reference, the anisotropic version of Pearl's vortex interaction for very thin film has been found. Their result is identical to Eq. (34).

${ }^{9}$ J.R. Kirtley, C.C. Tsuei, K.A. Moler, V.G. Kogan, J.R. Clem, and A.J. Turberfield, Appl. Phys. Lett. 74, 4011 (1999).

${ }^{10}$ G. Carneiro and E.H. Brandt, Phys. Rev. B 61, 6370 (2000); J.C. Wei and T.J. Yang, Jpn. J. Appl. Phys., Part 1 35, 5696 (1996). 